Verhaltenstherapie 1997;7:1-3

\title{
Content, Vol. 7, No. 1, 1997
}

\section{Inhalt $\cdot$ Contents}

Verhaltenstherapie

Vol. 7, Heft 1, März 1997

Vol. 7, No. 1, March 1997

\section{Editorial \\ 4 Editorial}

Hand, I. (Hamburg)

Originalarbeiten

Hand, I. (Hamburg)

Original Papers

5 Verhaltenstherapeutische Kurzzeit-

Gruppentherapie zur Versorgung von Patienten mit Angststörungen in der primärärztlichen

Praxis

Schulze, M; Osen, B.; Hand, I. (Hamburg)

14 Einzelfall-Versuchspläne: Veränderungs-Kriterium-Versuchsplan und Alternierender

Versuchsplan

Kern, H. J. (Oldenburg)

21 Rehabilitation von Aufmerksamkeitsstörungen nach einem Schlaganfall - Effektivität eines verhaltensmedizinisch-neuropsychologischen Aufmerksamkeitstrainings

Schöttke, H. (Osnabrück)

Diskussionsforum

5 Short-Term Group Therapy for Patients with Anxiety Disorders in Primary Care

Schulze, M.; Osen, B.; Hand, I. (Hamburg)

14 Single-Case Studies: Changing-Criterion Design and Alternating Design

Kern, H. J. (Oldenburg)

21 Rehabilitation of Attention Deficits after Stroke - Efficacy of a Neuropsychological Training

Program for Attention Deficits

Schöttke, H. (Osnabrück)

Forum

34 Einbeziehung der islamischen Religion in die kognitive Verhaltenstherapie in Malaysia Azhar, M. Z. (Kota Bharu, Malaysia)

Falldarstellung

34 Use of Islamic Religion in Psychotherapy in Malaysia

Azhar, M. Z. (Kota Bharu, Malaysia)

Case Report 
40 Kognitive Verhaltenstherapie bei einem 69jährigen depressiven Patienten

Soller, G. (Berlin)

48 Neuigkeiten vom Buch- und Zeitschriftenmarkt

40 Cognitive Behavior Therapy with a 69-Year-Old Depressive Patient

Soller, G. (Berlin)

48 Articles and Books

53 Mitteilungen der Verbände

53 Information by Behavioral Therapy Associations

Arbeitsgemeinsehaf't für Verhaltensmodifikation-Schweiz (AVM-CH)

Arbeitsgemeinsehaf't für Verhaltensmodifikation e. V. (AVM-D)

Centrum für Integrative Psychotherapie (CIP)

Deutsehe Gesellschaft für Sexualforschung

Deutscher Fachverband für Verhaltenstherapie (DVT)

Institut für Verhaltenstherapie e. V, Berlin

Vereinigung der Kassenpsychotherapeuten

60 Deutsche Gesellschaft für Verhaltenstherapie e. V.

61 Tagungen und Kongresse

61 Meetings and Conferences

2 Impressum U3 Hinweise für Autoren (3. Umschlagseite)

2 Imprint U3 Instructions to Authors (Inside back cover)

\section{KARGER}

(C) 1997 S. Karger GmbH. Freiburg Fax (0761)4520714 http://www.karger.ch

Editorial

Verhaltenstherapie

Verhaltenstherapie 1997;7:4

1. Hand

Editorial

Liebe Leserinnen, Hebe Leser, wieder geht VERHALTENSTHERAPIE in ein neues Lebens-jahr. Während die Nachfrage nach und das Angebot an Verhaltenstherapie im Lande weiter kontinuierlich zugenommen ha-ben, harren Probleme der Weiterbildung, der Kassenzulassung und der Qualitätssicherung teilweise immer noch der Lösung. Eine klare gesetzliche Regelung des Status der Psychologi-schen Verhaltenstherapeuten sowie die Präzisierung und Um-setzung der Weiterbildungsinhalte der verhaltenstherapeuti-schen Variante des neuen Facharztes Psychiatrie/Psychothera-pie müssen dringlichst einer Klärung zugeführt werden; die verhaltenstherapeutische Qualifizierung von Pflegepersonal in Psychiatrie und Medizin ist im Vergleich zu England und den USA immer noch auf wenige Kliniken beschränkt. Das GSG bleibt zweifellos eine Herausfordrung. Dennoch 
- die Weiterentwicklung und Akzeptanz unseres Faches ist, auch im internationalen Vergleich, 1996 wieder beeindruckend gewesen. Die genannten Probleme sind lösbar (s. Best, in diesem Heft), die standespolitischen Differenzen im Prinzip nicht unüberwindlich. Therapeutische Kreativität und forscherische Neugier haben ein immer breiter werden-des Arbeitsfeld, in dem Kooperation entscheidende Bedeu-tung behält.

Die Schriftleitung hat sich auf der letzten Sitzung im Herbst 96 bemüht, durch personelle Erweiterungen in Hauptschrift-leitung, Schriftleitung und wissenschaftlichem Beirat diese Entwicklungen aufzunehmen und das fachliche Kompetenz-spektrum entsprechend zu erweitern. Dadurch und durch Modifikationen in der Organisationsstruktur hoffen wir, die Motivation zur aktiven Mitgestaltung durch Beiträge und Akquisition von Beiträgen weiter zu fördern. Das Gremium der Hauptschriftleiter wurde von zwei auf vier Personen erweitert - mit einem Koordinator, der in bestimmten Abstän-den wechseln wird.

Neu in die Hauptschriftleitung gekommen sind Manfred Fichter, Dirk Hellhammer und Jürgen Margraf. Hans Witt-chen ist nach jahrelangem intensiven Einsatz auf seinen persönlichen Wunsch hin aus der Hauptschriftleitung in die Schriftleitung gewechselt. Als Schriftleiter neu hinzugekom-men sind Matthias Berger (Freiburg), Herta Flor (Berlin), Franz Petermann (Bremen), Winfried Rief (Prien), Dieter Vaitl (Gießen) und, im Wechsel, Hans-Ulrich Wittchen. Die aktuelle Zusammensetzung des Beirats ist aus dem Impres-sum zu ersehen. Mit dieser deutlichen Erweiterung des Spektrums der Fach-kompetenzen hoffen wir, noch besser als bisher die Verhaltenstherapie in Forschung, Lehre und Praxis in Deutschland abbilden und anregen zu können. Auch alle nicht in den Gremien unserer Zeitschrift vertretenen Kolleginnen und Kollegen möchten wir bei dieser Gelegenheit wieder herzlich zur aktiven Mitarbeit einladen. Im Rahmen der Neuerungen möchten wir ein Procedere aus dem ersten Jahrgang unserer Zeitschrift neu beleben: Das Einholen von eingeladenen Beiträgen durch einzelne Hauptschriftleiter. Diese Beiträge gehen nicht mehr in das Review-Verfahren, die inhaltliche Verantwortung liegt allein beim Autor und dem einladenden Hauptschriftleiter (s. Azhar, in diesem Heft).

Hans-Ulrich Wittchen und seiner Mitarbeiterin, Gerlinde Winter, gilt auch an dieser Stelle der herzliche Dank der gesamten Schriftleitung, des Beirats und des Verlages für die in den Aufbaujahren der Zeitschrift engagiert und äußerst erfolgreich geleistete Koordinations- und Akquisitionsarbeit. Sie haben beeindruckende Standards geschaffen, auf denen wir mit Ihrer aller Anregungen und Hilfe weiter aufbauen können.

Mit alien guten Wünschen für 1997 von Schriftleitung, Beirat und Verlag.

\section{KARGER}

(C) 1997 S. Karger GmbH. Freiburg Fax (0761) 4520714 http://www.karger.ch

Prof. Dr. Iver Hand

Universitäts-Krankenhavs Eppendorf

Psychiatrische und Nervenklinik

Verhaltenstherapie-Ambulanz

M. Martinistraße 52

D-20246 Hamburg (Deutschland) 